\title{
Formation of carbon grains in the atmosphere of IRC+10216
}

\section{The role of periodic shocks in the formation of PAHs and their dimers}

\author{
P. $\mathrm{Cau}^{\star}$ \\ 1 Department of Physics, UMIST, PO Box 88, Manchester M60 1QD, UK \\ ${ }^{2}$ LUTH, Observatoire de Paris-Meudon, 92195 Meudon, France
}

Received 4 September 2001 / Accepted 17 June 2002

\begin{abstract}
We investigate the contribution of periodic shocks to the formation of polycyclic aromatic hydrocarbons (PAHs) and their dimers in the inner atmosphere of IRC+10216. The goal of this paper is to look at the implications this has for the formation of carbon dust if PAHs are an intermediate step. It is shown that the amount of PAHs and dimers produced is not enough to explain the formation of carbon dust in the atmosphere of the star, but it can account for the formation of the small disordered cores of the kind observed in presolar grains.
\end{abstract}

Key words. stars: circumstellar matter - stars: AGB - stars: individual: IRC+10216

\section{Introduction}

In this paper we investigate the role of periodic shocks in the formation of PAHs and their dimers in the atmosphere of the carbon rich AGB star IRC+10216. Chemical models of the formation of PAHs in acetylene flames have been successfully developed by Frenklach and coworkers (e.g. Frenklach et al. $1985,1986 a, b)$ and they are of paramount interest for the astrophysicists who want to test the same chemistry in space. The only major difference between an acetylene flame and the circumstellar envelope of a carbon-rich AGB star is the presence of oxygen in the former, which is absent in the latter, where it is locked in the very stable CO molecule. Frenklach \& Feigelson (1989) and Cherchneff et al. (1992) have applied the chemistry of PAH formation to the circumstellar envelopes of carbon-rich AGB stars. In particular they found that the production of PAHs is efficient only when the temperature of the gas is between 900 and $1100 \mathrm{~K}$. A similar result was found by Helling et al. (1996), who used equilibrium calculations to show that the production of PAHs can be efficient when the gas temperature is in the window 700-900 K, provided the gas pressure is substantially higher than usually assumed in standard wind models.

PAHs are thought to be an intermediate step towards the formation of carbon grains, and an important test for these models is to compare the amount of carbon that "migrates" into PAHs with the amount of carbon that is known to be in the form of dust. All the investigations above have shown that the former is invariably lower than the latter. However, they used a simple, steady wind model, and this raised the question if it is precisely

^ e-mail: pierocau@hotmail.com the poor wind model which is the reason for the failure of the calculations in predicting the formation of PAH molecules in an amount comparable in mass with that of circumstellar grains.

Here we address this question using an improved model of the stellar wind (Fox \& Wood 1986; Bowen 1988; Willacy \& Cherchneff 1998) which takes into account the effect of periodic shocks which are known to be present in the inner wind of AGB stars (e.g. Gillet et al. 1983). Such shocks induce a periodic variation of temperature and density in the gas. As a result, a given parcel of gas passes through the temperature window of efficient PAH formation more than one time. Since PAHs are quite stable, it is possible that the periodic, repeated passage of the gas in the critical window could allow significant amounts of PAHs and their dimers to form.

\section{The model}

The presence of shock waves has two important consequences (e.g. Bowen 1988): (i) the gas is lifted upwards by the shocks, and this fact produces an extended zone in the atmosphere in which the concentration is higher than it would be if the shocks were not taking place, and (ii) during each period, parcels of gas are accelerated upwards by the shocks and fall back under stellar gravity; during these excursions, concentration and temperature of the gas change dramatically. To model this we adopt a semi-analytical method first developed by Bertschinger \& Chevalier (1985). The implementation we adopt has been first used by Willacy \& Cherchneff (1998). The details of it are given in the Appendix. 
Table 1. Preshock, shock front and excursion gas temperature and number concentration for the various shock velocities $v_{\text {shock }}$ chosen in our model.

\begin{tabular}{ccccccccc}
\hline \hline$v_{\text {shock }}$ & Radius & Mach & \multicolumn{2}{c}{ Preshock } & \multicolumn{2}{c}{ Shock Front } & \multicolumn{2}{c}{ Start of Excursion } \\
$\left(\mathrm{km} \mathrm{s}^{-1}\right)$ & $\left(R_{*}\right)$ & number & $T_{0}(\mathrm{~K})$ & $n_{0}\left(\mathrm{~cm}^{-3}\right)$ & $T(\mathrm{~K})$ & $n\left(\mathrm{~cm}^{-3}\right)$ & $T_{\mathrm{c} / \mathrm{h}}(\mathrm{K})$ & $n_{\mathrm{c} / \mathrm{h}}\left(\mathrm{cm}^{-3}\right)$ \\
\hline 18.5 & 1.4 & 6.46 & 1879 & $1.36 \times 10^{14}$ & 17016 & $7.29 \times 10^{14}$ & 4214 & $2.30 \times 10^{15}$ \\
16.8 & 1.7 & 6.21 & 1673 & $4.23 \times 10^{13}$ & 14118 & $2.25 \times 10^{14}$ & 3656 & $6.94 \times 10^{14}$ \\
15.9 & 1.9 & 6.07 & 1565 & $2.25 \times 10^{13}$ & 12646 & $1.19 \times 10^{14}$ & 3395 & $3.61 \times 10^{14}$ \\
14.1 & 2.4 & 5.80 & 1360 & $6.56 \times 10^{12}$ & 10176 & $3.43 \times 10^{13}$ & 2861 & $9.79 \times 10^{13}$ \\
12.6 & 3.0 & 5.54 & 1190 & $2.24 \times 10^{12}$ & 8218 & $1.16 \times 10^{13}$ & 2441 & $3.11 \times 10^{13}$ \\
\hline
\end{tabular}
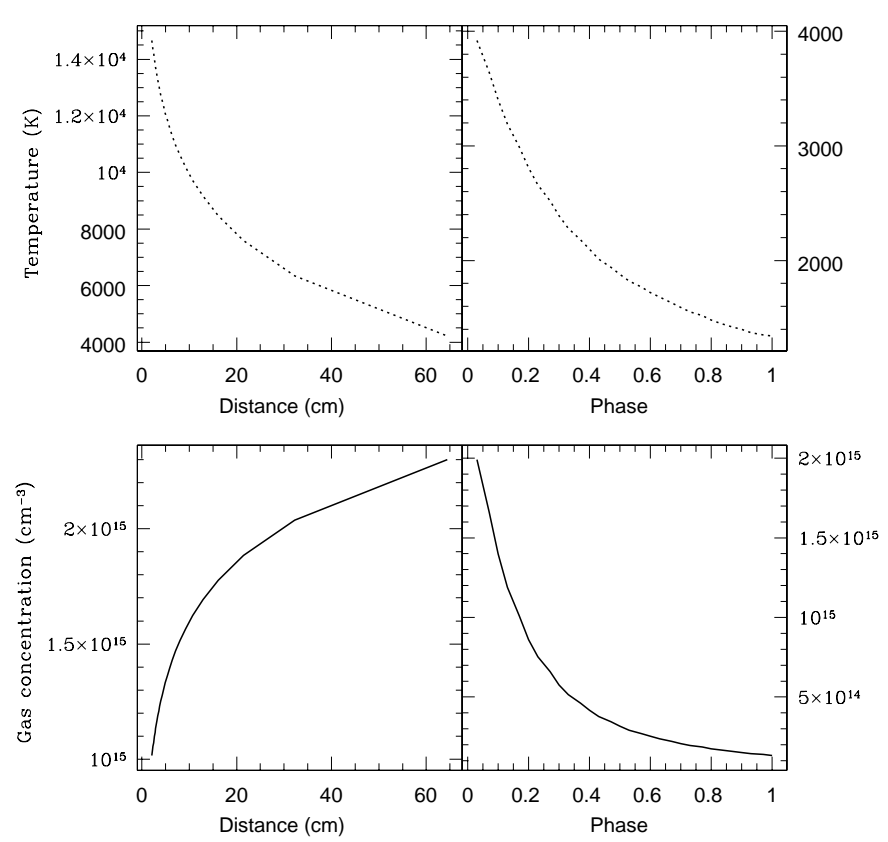

Fig. 1. Temperature and concentration profile of the gas at $1.4 R_{*}$ in the chemical cooling region and during the hydrodynamic cooling (expansion).

We consider the inner circumstellar envelope (CSE) as a narrow region above the photosphere which experiences the passage of periodic shocks generated by the stellar pulsations. We assume that shocks form at $1.2 R_{*}$ with an initial velocity of $20 \mathrm{~km} \mathrm{~s}^{-1}$ and that they are damped as they travel outward. The choice of the shock formation radius $r_{\text {shock }}$ as well as of its initial velocity is arbitrary but reflects typical values found in carbon stars. We consider five different distances from the center of star $\left(1.4 R_{*}, 1.7 R_{*}, 1.9 R_{*}, 2.4 R_{*}\right.$ and $\left.3 R_{*}\right)$ and follow the chemistry of a parcel of gas starting when it is reached by a shock to the moment in which the next shock arrives. During the excursion the parcel moves upwards, but we assume that under stellar gravity it comes back to its initial position (see the Appendix). At each distance the shock is assumed to be plane-parallel. Table 1 reports the different shock velocities at the various distances, together with temperatures and concentration of the gas before the shocks reach it, immediately behind the shock front and at the beginning of the adiabatic expansion (see the Appendix).

In Figs. 1 and 2 we plot the profile of concentration and temperature of a parcel of gas at $1.4 R_{*}$ and $3 R_{*}$ during an
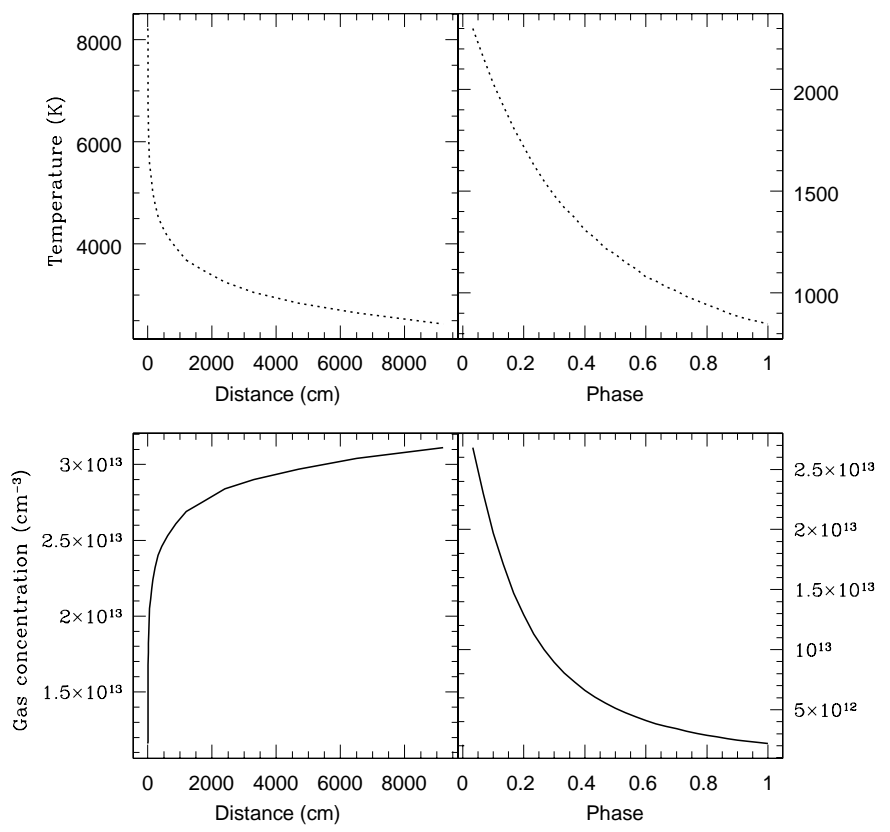

Fig. 2. Temperature and concentration profile of the gas at $3 R_{*}$ in the chemical cooling region and during the hydrodynamic cooling (expansion).

entire excursion. The system of reference of the shock is adopted, so what the figures show on the left is the temperature and concentration of a given parcel of gas while it moves away from the shock front. The cooling here is dominated by the dissociation of molecular hydrogen. Another cooling process, the adiabatic expansion of the gas, becomes more efficient at lower temperature. On the right, the figures show the temperature and concentration vs. phase (= time/period) during this second stage. At the end of the expansion (phase =1), the parcel reaches its initial temperature and density, it passes through another shock front and the cycle repeats itself. More details can be found in the Appendix.

\section{Model star}

Our model is applied to IRC+10216 (= AFGL $1381=\mathrm{CW}$ Leo). The adopted parameters for this star are listed in Table 2. Besides being the best studied carbon star, its physical conditions match with the requirements of our model, as discussed in Sect. A.4 of the Appendix. 
Table 2. The stellar parameters assumed for IRC +10216 . (From Willacy \& Cherchneff 1998.)

\begin{tabular}{ll}
\hline \hline Temperature & $2300 \mathrm{~K}$ \\
Luminosity & $2.5 \times 10^{4} L_{\odot}$ \\
Radius & $7.0 \times 10^{13} \mathrm{~cm}$ \\
Mass & $2 M_{\odot}$ \\
Period & 650 days \\
Concentration $\left(1.2 R_{*}\right)$ & $3.7 \times 10^{14} \mathrm{~cm}^{-3}$ \\
$\mathrm{C} / \mathrm{O}$ & 1.5 \\
\hline
\end{tabular}

\section{PAH condensation sequence}

We adopt the reaction network of Frenklach \& Feigelson (1989) as improved by Cherchneff et al. (1992). This network describes the formation of PAHs from acetylene and hydrogen. Various chemical paths leading to the formation of benzene and naphtalene are considered. Given the lack of information on the following growth of PAHs, it is assumed that this proceeds through analogous reactions leading to the formation of the first aromatic ring, through repeated abstraction of $\mathrm{H}$ atoms and the addition of acetylene. This mechanism of growth, also known as linear lumping, is appropriate to describe the formation of catacondensed PAHs. In order to study the formation of pericondensed PAHs such as pyrene and coronene, Allain et al. (1997) modified the reaction network so that the formation of pericondensed species is achieved through the addition of a single acetylene molecule. We do not introduce this modification for two reasons. First, we need to estimate the contribution of periodic shocks by comparing our results with those of other authors who used the same chemistry but did not consider the contribution of the shocks, such as Frenklach \& Feigelson (1989) and Cherchneff et al. (1992). Second, given the present lack of knowledge on the details of the chemistry of PAHs, we avoid any conclusion on the abundance of any PAHs considered. Rather, we use our results to evaluate how efficiently carbon is converted from simple hydrocarbons into benzene and PAHs, and discuss the implications of this efficiency for the formation of carbon dust in CSEs.

In this work we extend the chemical scheme of Cherchneff et al. (1992) to include PAHs with up to seven aromatic rings as in Cherchneff (1997). We also consider the formation of dimers of PAHs as discussed in Cherchneff (1997). The rate of each reaction is given in Cherchneff et al. (1992). For PAHs not considered by Cherchneff et al. (1992) the rates of forward reactions are the same as analogous reactions involving smaller PAHs. The rates for the reverse reactions are estimated using the thermodynamic properties of the molecules involved (namely, the Gibbs free energy of formation) and are given by (Eq. (44) in Cherchneff et al. 1992)

$k_{\mathrm{r}}=A T^{n} C^{\Delta v} \exp \left(\frac{1000}{R}\left[\frac{G_{1}^{\mathrm{r}}-E_{\mathrm{a}}}{T}+G_{2}^{\mathrm{r}}+G_{3}^{\mathrm{r}} T+G_{4}^{\mathrm{r}} T^{2}+G_{5}^{\mathrm{r}} T^{3}\right]\right)$

where $A, n$ and $E_{\mathrm{a}}$ are the Arrhenius parameters of the forward reaction, $T$ is the kinetic temperature of the gas (in $\mathrm{K}$ ), $\Delta v$ is the change in the number of molecules due to the reaction and the $G_{i}^{\mathrm{r}} \mathrm{s}$ are the fitting coefficients of the Gibbs free energy
$(\mathrm{kJ} /$ mole) of the reaction, given by the difference between the total Gibbs free energy of formation of the products and that of the reactants in the forward reaction. These energies are given in the JANAF Tables (Chase et al. 1985) for various temperatures, and Cherchneff et al. (1992) used a polynomial of fourth order to fit them. As the values tabulated in the JANAF Tables are given for a gas at the pressure of 1 atmosphere, the factor $C=\frac{N_{\mathrm{A}} \times 10^{6}}{R T}$ converts the units into $\mathrm{cm}^{-3}$. Here $N_{\mathrm{A}}$ is the Avogadro number and $R$ is the perfect gas constant.

The values of the coefficients of the polynomial fitting of the Gibbs free energy of formation for the species not included in Cherchneff et al. (1992) are given in Table 3 (courtesy of Isabelle Cherchneff). They are estimated according to the group additivity method (Stein \& Fahr 1985).

\section{Formation of dimers of PAHs}

We consider the formation of dimers of PAHs formed by, at most, two PAHs with seven aromatic rings each. Following Cherchneff (1997) we assume that the dimers are formed through the reaction of PAHs having the same number of aromatic rings. The reaction rates for dimer formation from their aromatic parents has been measured (Fahr et al. 1988) only for the reaction of benzene with phenyl

$\mathrm{C}_{6} \mathrm{H}_{6}+\mathrm{C}_{6} \mathrm{H}_{5} \rightarrow \mathrm{C}_{6} \mathrm{H}_{5} \mathrm{C}_{6} \mathrm{H}_{5}+\mathrm{H}$.

For larger dimers we follow Cherchneff (1997) who considered that the van der Waals force between molecules acts as a temporary glue which holds the adduct together while the chemical bond forms. This force increases with the size of molecules, and so should the rate coefficient. Miller et al. (1984) have estimated the van der Waals forces for several PAHs and their results have been used by Cherchneff (1997) to scale the reaction rates with respect to the force values.

The rate of each reaction leading to the formation of dimers is of the form

$A \exp \left(\frac{-16720 \mathrm{~J}^{\mathrm{mole}} \mathrm{e}^{-1}}{R T}\right)$

where the values of $A$ for each reaction are given in Table 4 $\left(\mathrm{DA}_{n}\right.$ represents a dimer composed of two PAHs with $n$ aromatic rings, linked together by a chemical bond).

The destruction of $\mathrm{C}_{6} \mathrm{H}_{5} \mathrm{C}_{6} \mathrm{H}_{5}$ leading to two $\mathrm{C}_{6} \mathrm{H}_{5}$

$\mathrm{C}_{6} \mathrm{H}_{5} \mathrm{C}_{6} \mathrm{H}_{5} \rightarrow \mathrm{C}_{6} \mathrm{H}_{5}+\mathrm{C}_{6} \mathrm{H}_{5}$

is described by Cherchneff (1997) with the rate of $1.6 \times 10^{-38} T^{5}$ $\exp \left(-2400 \mathrm{~J}\right.$ mole $\left.\mathrm{e}^{-1} / R T\right) \mathrm{s}^{-1}$, and the activation energy in the exponent is rescaled for bigger dimers according to their size. The value $E_{\mathrm{a}}$ to replace $2400 \mathrm{~J}$ mole ${ }^{-1}$ in the rate, for each dimer, is reported in the third column of Table 4.

\section{Temperature of PAHs}

As first noted by Cherchneff et al. (1991) the vibrational temperature of PAHs can be different from the kinetic temperature of the gas. This is because if the density of the gas is low enough, collisions are not efficient in coupling the vibrational 
Table 3. Coefficients of the Gibbs free energy of formation in $\mathrm{kJ} / \mathrm{mole}$, at 1 atmosphere. Values not included in Cherchneff et al. (1992). (Courtesy of Isabelle Cherchneff.)

\begin{tabular}{|c|c|c|c|c|c|}
\hline Species & $\begin{array}{c}G_{1}^{f} \\
\mathrm{~kJ} \mathrm{~mole}^{-1}\end{array}$ & $\begin{array}{c}G_{2}^{f} \\
\mathrm{~kJ} \mathrm{~mole}^{-1} \mathrm{~K}^{-1}\end{array}$ & $\begin{array}{c}G_{3}^{f} \\
\mathrm{~kJ} \mathrm{~mole}^{-1} \mathrm{~K}^{-2}\end{array}$ & $\begin{array}{c}G_{4}^{f} \\
\mathrm{~kJ} \mathrm{~mole}^{-1} \mathrm{~K}^{-3}\end{array}$ & $\begin{array}{c}G_{5}^{f} \\
\mathrm{~kJ} \mathrm{~mole}^{-1} \mathrm{~K}^{-4}\end{array}$ \\
\hline $\mathrm{A}_{3^{-}}$ & $4.51 \times 10^{+02}$ & $2.56 \times 10^{-01}$ & $2.84 \times 10^{-05}$ & $-1.01 \times 10^{-08}$ & $8.70 \times 10^{-13}$ \\
\hline $\mathrm{A}_{3}$ & $2.04 \times 10^{+02}$ & $3.30 \times 10^{-01}$ & $4.17 \times 10^{-05}$ & $-1.25 \times 10^{-08}$ & $1.08 \times 10^{-12}$ \\
\hline $\mathrm{A}_{3} \mathrm{C}_{2} \mathrm{H}_{2} \#$ & 0. & 0. & 0. & 0. & 0. \\
\hline $\mathrm{A}_{3} \mathrm{C}_{2} \mathrm{H}$ & $4.41 \times 10^{+02}$ & $2.76 \times 10^{-01}$ & $5.79 \times 10^{-05}$ & $-1.97 \times 10^{-08}$ & $1.71 \times 10^{-12}$ \\
\hline $\mathrm{A}_{3} \mathrm{C}_{2} \mathrm{H}_{2} \mathrm{U}$ & $5.20 \times 10^{+02}$ & $3.21 \times 10^{-01}$ & $4.80 \times 10^{-05}$ & $-1.96 \times 10^{-08}$ & $1.70 \times 10^{-12}$ \\
\hline $\mathrm{A}_{3} \mathrm{C}_{2} \mathrm{H}$ & $6.88 \times 10^{+02}$ & $2.08 \times 10^{-01}$ & $4.46 \times 10^{-05}$ & $-1.73 \times 10^{-08}$ & $1.49 \times 10^{-12}$ \\
\hline $\mathrm{A}_{4^{-}}$ & $4.72 \times 10^{+02}$ & $2.47 \times 10^{-01}$ & $4.78 \times 10^{-05}$ & $-1.84 \times 10^{-08}$ & $1.61 \times 10^{-12}$ \\
\hline $\mathrm{A}_{4}$ & $2.24 \times 10^{+02}$ & $3.18 \times 10^{-01}$ & $7.82 \times 10^{-05}$ & $-3.25 \times 10^{-08}$ & $2.85 \times 10^{-12}$ \\
\hline $\mathrm{A}_{4} \mathrm{C}_{2} \mathrm{H}_{2} \#$ & 0. & 0. & 0. & 0 . & 0 . \\
\hline $\mathrm{A}_{4} \mathrm{C}_{2} \mathrm{H}$ & $4.53 \times 10^{+02}$ & $2.73 \times 10^{-01}$ & $7.61 \times 10^{-05}$ & $-3.16 \times 10^{-08}$ & $2.75 \times 10^{-12}$ \\
\hline $\mathrm{A}_{4} \mathrm{C}_{2} \mathrm{H}_{2} \mathrm{U}$ & $5.32 \times 10^{+02}$ & $3.12 \times 10^{-01}$ & $6.60 \times 10^{-05}$ & $-3.20 \times 10^{-08}$ & $2.79 \times 10^{-12}$ \\
\hline $\mathrm{A}_{4} \mathrm{C}_{2} \mathrm{H}$ & $7.00 \times 10^{+02}$ & $2.04 \times 10^{-01}$ & $6.28 \times 10^{-05}$ & $-2.92 \times 10^{-08}$ & $2.54 \times 10^{-12}$ \\
\hline $\mathrm{A}_{5^{-}}$ & $5.11 \times 10^{+02}$ & $2.88 \times 10^{-01}$ & $7.34 \times 10^{-05}$ & $-3.37 \times 10^{-08}$ & $2.95 \times 10^{-12}$ \\
\hline $\mathrm{A}_{5}$ & $2.64 \times 10^{+02}$ & $3.62 \times 10^{-01}$ & $8.67 \times 10^{-05}$ & $-3.61 \times 10^{-08}$ & $3.16 \times 10^{-12}$ \\
\hline $\mathrm{A}_{5} \mathrm{C}_{2} \mathrm{H}_{2} \#$ & 0. & 0. & 0. & 0. & 0. \\
\hline $\mathrm{A}_{5} \mathrm{C}_{2} \mathrm{H}$ & $4.93 \times 10^{+02}$ & $3.22 \times 10^{-01}$ & $8.38 \times 10^{-05}$ & $-3.52 \times 10^{-08}$ & $3.06 \times 10^{-12}$ \\
\hline $\mathrm{A}_{5} \mathrm{C}_{2} \mathrm{H}_{2} \mathrm{U}$ & $5.72 \times 10^{+02}$ & $3.61 \times 10^{-01}$ & $7.38 \times 10^{-05}$ & $-3.56 \times 10^{-08}$ & $3.10 \times 10^{-12}$ \\
\hline $\mathrm{A}_{5} \mathrm{C}_{2} \mathrm{H}$ & $7.40 \times 10^{+02}$ & $2.54 \times 10^{-01}$ & $7.05 \times 10^{-05}$ & $-3.28 \times 10^{-08}$ & $2.85 \times 10^{-12}$ \\
\hline $\mathrm{A}_{6^{-}}$ & $5.51 \times 10^{+02}$ & $3.31 \times 10^{-01}$ & $8.20 \times 10^{-05}$ & $-3.78 \times 10^{-08}$ & $3.30 \times 10^{-12}$ \\
\hline $\mathrm{A}_{6}$ & $3.04 \times 10^{+02}$ & $4.05 \times 10^{-01}$ & $9.54 \times 10^{-05}$ & $-4.02 \times 10^{-08}$ & $3.52 \times 10^{-12}$ \\
\hline $2 \mathrm{~A}_{6} \mathrm{C}_{2} \mathrm{H}_{2} \#$ & 0. & 0. & 0. & 0 . & 0. \\
\hline $\mathrm{A}_{6} \mathrm{C}_{2} \mathrm{H}$ & $5.31 \times 10^{+02}$ & $3.74 \times 10^{-01}$ & $5.56 \times 10^{-05}$ & $-3.30 \times 10^{-08}$ & $2.86 \times 10^{-12}$ \\
\hline $\mathrm{A}_{6} \mathrm{C}_{2} \mathrm{H}_{2} \mathrm{U}$ & $6.09 \times 10^{+02}$ & $4.17 \times 10^{-01}$ & $3.73 \times 10^{-05}$ & $-3.34 \times 10^{-08}$ & $2.90 \times 10^{-12}$ \\
\hline $\mathrm{A}_{6} \mathrm{C}_{2} \mathrm{H}$ & $7.78 \times 10^{+02}$ & $3.05 \times 10^{-01}$ & $4.23 \times 10^{-05}$ & $-3.06 \times 10^{-08}$ & $2.65 \times 10^{-12}$ \\
\hline$A_{7-}$ & $5.64 \times 10^{+02}$ & $3.25 \times 10^{-01}$ & $8.16 \times 10^{-05}$ & $-3.85 \times 10^{-08}$ & $3.36 \times 10^{-12}$ \\
\hline $\mathrm{A}_{7}$ & $3.17 \times 10^{+02}$ & $3.05 \times 10^{-01}$ & $4.23 \times 10^{-05}$ & $-3.06 \times 10^{-08}$ & $2.65 \times 10^{-12}$ \\
\hline
\end{tabular}

Table 4. Rate parameters $A$ for the formation and $E_{\mathrm{a}}$ for the destruction of dimers. (Courtesy of Isabelle Cherchneff.)

\begin{tabular}{lcc}
\hline \hline Dimer & $A\left(\mathrm{~cm}^{3} \mathrm{~s}^{-1}\right)$ & $E_{\mathrm{a}}\left(\mathrm{J} \mathrm{mole}^{-1}\right)$ \\
\hline $\mathrm{DA}_{1}$ & $6.64 \times 10^{-13}$ & $2.40 \times 10^{3}$ \\
$\mathrm{DA}_{2}$ & $1.07 \times 10^{-12}$ & $3.85 \times 10^{3}$ \\
$\mathrm{DA}_{3}$ & $1.49 \times 10^{-12}$ & $5.39 \times 10^{3}$ \\
$\mathrm{DA}_{4}$ & $1.71 \times 10^{-12}$ & $6.16 \times 10^{3}$ \\
$\mathrm{DA}_{5}$ & $2.02 \times 10^{-12}$ & $7.31 \times 10^{3}$ \\
$\mathrm{DA}_{6}$ & $2.34 \times 10^{-12}$ & $8.47 \times 10^{3}$ \\
$\mathrm{DA}_{7}$ & $2.18 \times 10^{-12}$ & $7.90 \times 10^{3}$ \\
$\mathrm{DA}_{2} \mathrm{R}_{5}$ & $1.28 \times 10^{-12}$ & $4.62 \times 10^{3}$ \\
$\mathrm{DA}_{3} \mathrm{R}_{5}$ & $1.60 \times 10^{-12}$ & $6.16 \times 10^{3}$ \\
$\mathrm{DA}_{4} \mathrm{R}_{5}$ & $1.92 \times 10^{-12}$ & $6.93 \times 10^{3}$ \\
\hline
\end{tabular}

and translational degrees of freedom, so that the vibrational temperature can be significantly lower than the kinetic one. To estimate this effect on PAHs during the passage of periodic shocks, we solve Eq. (45) in Cherchneff et al. (1992)

$n T^{1.5}-n T^{0.5} T_{\mathrm{PAH}}=\Omega T_{\mathrm{PAH}}^{B+4}$

for the density and temperature profile of the shocks. Here $n$ and $T$ are the number density and kinetic temperature of the
Table 5. Parameters $A, B$ and $a$ used in Eq. (5).

\begin{tabular}{lccc}
\hline \hline PAH & $A$ & $B$ & $a(\mathrm{~cm})$ \\
\hline $\mathrm{A}_{1}$ & $1.31 \times 10^{-15}$ & -1.73 & $2 \times 10^{-8}$ \\
$\mathrm{~A}_{2}$ & $9.10 \times 10^{-15}$ & -1.86 & $2.8 \times 10^{-8}$ \\
$\mathrm{~A}_{3}$ & $9.10 \times 10^{-15}$ & -1.78 & $3.46 \times 10^{-8}$ \\
$\mathrm{~A}_{4}$ & $1.60 \times 10^{-15}$ & -1.47 & $4 \times 10^{-8}$ \\
$\mathrm{~A}_{5}$ & $5.22 \times 10^{-15}$ & -1.64 & $4.47 \times 10^{-8}$ \\
$\mathrm{~A}_{6}$ & $4.29 \times 10^{-15}$ & -1.58 & $4.88 \times 10^{-8}$ \\
$\mathrm{~A}_{7}$ & $4.97 \times 10^{-15}$ & -1.58 & $5.27 \times 10^{-8}$ \\
\hline
\end{tabular}

gas, $T_{\mathrm{PAH}}$ is the temperature of the considered PAH and $\Omega=$ $2.2776 \times 10^{8}[A / a]$ where $a$ is the PAH molecular radius. The two parameters $A$ and $B$ are used to represent the Planck mean of extinction cross section and are given in Cherchneff et al. (1992). We report the values of $A, B$ and $a$ in Table 5 .

We plot the results at $3 R_{*}$ in Fig. 3. Since the temperature of the gas and that of PAHs turn out to be very close to each other, to show their relation we plot the relative value of the latter with respect to the former $\left(T_{\mathrm{PAH}} / T_{\mathrm{gas}}\right)$.

The solution of Eq. (5) shows that while the difference between the two is more than $5 \%$ at $3 R_{*}$ (Fig. 3), it reduces to $2-3 \%$ at $2.4 R_{*}$, to $1 \%$ at $1.7 R_{*}$ and to less than $1 \%$ at $1.4 R_{*}$. 


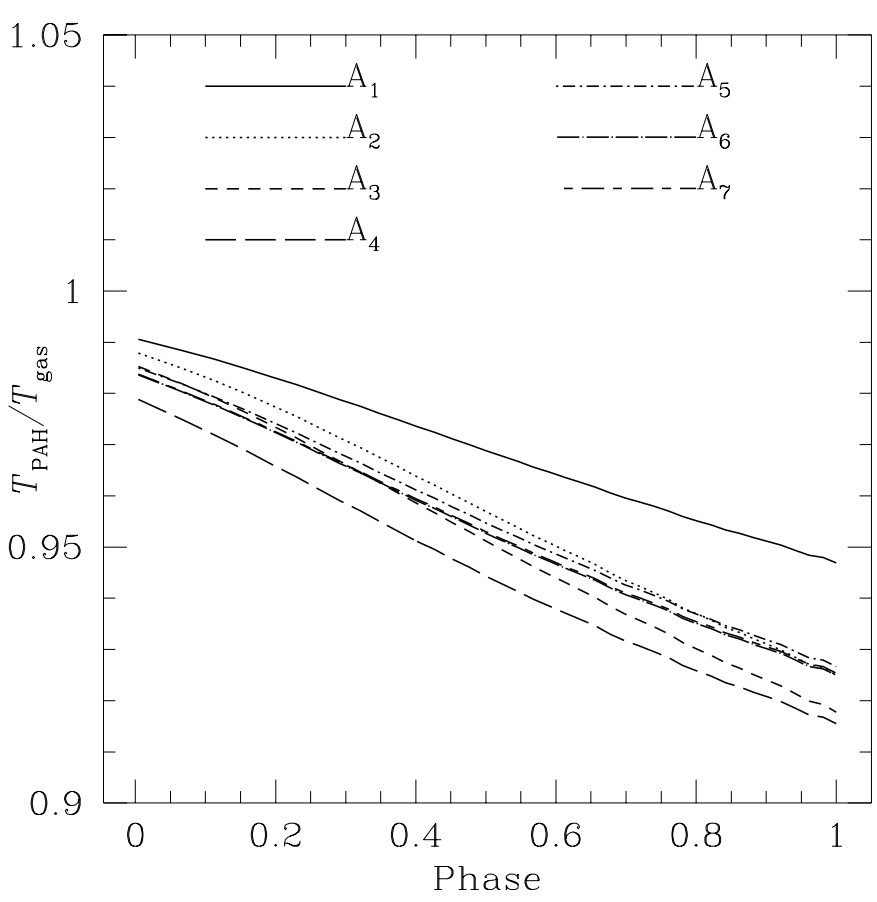

Fig. 3. Inverse greenhouse effect during the excursion at $3 R_{*}$.

In our calculation we consider the difference between the two temperatures only at $3 R_{*}$.

\section{Model}

We have used Eq. (1) to determine the rate of reverse reactions, adopting the Gibbs coefficients computed by Cherchneff et al. (1992). Such fittings are not appropriate for very high temperature, when some of the resulting rates become very high. For this reason we replace such "thermodynamic" rates with a collisional rate each time the latter is smaller.

The collisional rate for a reaction

$\mathrm{A}+\mathrm{B} \rightarrow \mathrm{C}+\mathrm{D}$

is given by (e.g. Avery 1974)

$n_{\mathrm{A}} n_{\mathrm{B}} \sigma_{\mathrm{AB}}^{2}\left(\frac{8 \pi R T}{\mu}\right)^{\frac{1}{2}}$

where $n_{\mathrm{A}}$ and $n_{\mathrm{B}}$ are the concentrations of the species $\mathrm{A}$ and $\mathrm{B}$, $\mu$ is the reduced molar mass defined as

$\mu=\frac{M_{\mathrm{A}} M_{\mathrm{B}}}{M_{\mathrm{A}}+M_{\mathrm{B}}}$

where $M_{\mathrm{A}}$ and $M_{\mathrm{B}}$ are the molar masses of species A and $\mathrm{B}$, and $\sigma_{\mathrm{AB}}$ is the mean collisional diameter defined by

$\sigma_{\mathrm{AB}}=\frac{\sigma_{\mathrm{A}}+\sigma_{\mathrm{B}}}{2}$

where $\sigma_{\mathrm{A}}$ and $\sigma_{\mathrm{B}}$ are the collisional diameters of species A and B. $R$ is the gas constant.

Rather than defining a collisional rate for each reaction, we choose to consider a "standard" rate and apply it to all the reverse reactions each time the rate determined using thermodynamic considerations is higher. To determine this "standard" rate, we consider molecules with the same properties as benzene. The collisional diameter of benzene, assuming its geometrical value, is $4 \AA$ (see also Table 5 which reports the radius $a$ of all the PAHs included in the model) and the reduced molar mass is $(6 \times 12 \mathrm{~g}) / 2$, where we have ignored hydrogen atoms. Using cgs units we obtain

$n_{\mathrm{A}} n_{\mathrm{B}}\left(4 \times 10^{-8}\right)^{2}\left(\frac{8 \pi 8.31 \times 10^{7} T}{36}\right)^{\frac{1}{2}}=1.3 \times 10^{-11} T^{\frac{1}{2}} n_{\mathrm{A}} n_{\mathrm{B}}$.

This collisional rate will be used for the reverse reactions each time that the corresponding thermodynamic rate is larger.

\section{Calculations and results}

\subsection{Initial concentrations}

The full list of the chemical species included in our model is shown in Table 6. The formalism of Frenklach \& Feigelson (1989) is adopted. In particular $\mathrm{A}_{i}$ denotes a PAH with $i$ aromatic rings. Note that the concentration of $\mathrm{A}_{i}$ refers to the concentration of chemically similar (in the context of PAH growth) compounds (see Frenklach \& Feigelson 1989).

We first perform an integration starting at $1.4 R_{*}$ during the hydrodynamic cooling. After the first integration which covers one pulsational period the resulting concentrations are rescaled to the concentration of the gas at the beginning of the hydrodynamic cooling and a new integration is performed over the next pulsational period. At $1.4 R_{*}$ we ignore the chemical cooling which does not last enough for any significant chemistry to take place. Performing further integrations in the same way turns out not to change significantly the output of the integration, so that the output after two pulsational periods is used as input at the beginning of the chemical cooling at $1.7 R_{*}$, after rescaling the concentration of all species to that of the gas at $1.7 R_{*}$.

At $1.7 R_{*}$ (as well as at further distances) we consider the chemical cooling. Each cycle of integration, corresponding to a pulsational period, is composed by a first stage dominated by the chemical cooling and a second stage dominated by the hydrodynamic cooling. The concentrations resulting from the first stage are used as input for the second stage, and two cycle are performed. Again, performing further integrations turns out not to change the output significantly, so the output of the second cycle is rescaled to the concentration of the gas at the next distance and two cycles of integration are performed at the next distance. This is done up to $3 R_{*}$, the outer distance considered in our model.

To determine the values of the initial concentrations, we repeat the calculations of Willacy \& Cherchneff (1998) whose chemical network includes all the hydrocarbons that are relevant in our model. Because of the high density and temperature, the chemical composition of the photosphere can be derived by chemical equilibrium calculations. We performed these calculations using the computer program CHEMEQ written by Andrew Markwick, which implements the method described by Sharp \& Huebner (1990) and determines the concentration 
Table 6. List of all chemical species included in our model. The formalism is that of Frenklach \& Feigelson (1989), also used by Cherchneff et al. (1992).

\begin{tabular}{|c|c|c|c|c|}
\hline$\overline{\mathrm{H}}$ & $\overline{\mathrm{C}_{6} \mathrm{H}_{7} \mathrm{U}}$ & $\overline{\mathrm{A}_{3} \mathrm{R}_{5} \mathrm{C}_{2} \mathrm{H}_{2} \#}$ & $\overline{\mathrm{A}_{4} \mathrm{C}_{2} \mathrm{H}}$ & $\overline{\mathrm{CH}}$ \\
\hline $\mathrm{H}_{2}$ & $\mathrm{C}_{6} \mathrm{H}_{5} \mathrm{U}$ & $\mathrm{A}_{3} \mathrm{R}_{5} \mathrm{C}_{2} \mathrm{H}$ & $\mathrm{A}_{4} \mathrm{C}_{2} \mathrm{H}_{2} \mathrm{U}$ & $\mathrm{C}_{3} \mathrm{H}$ \\
\hline $\mathrm{C}_{2} \mathrm{H}_{2}$ & $\mathrm{C}_{6} \mathrm{H}_{3} \mathrm{U}$ & $\mathrm{A}_{3} \mathrm{R}_{5} \mathrm{C}_{2} \mathrm{H}_{2} \mathrm{U}$ & $\mathrm{A}_{4} \mathrm{C}_{2} \mathrm{H}^{*}$ & $\mathrm{DA}_{1}$ \\
\hline $\mathrm{C}_{2} \mathrm{H}$ & $\mathrm{C}_{6} \mathrm{H}_{6}$ & $\mathrm{~A}_{3} \mathrm{R}_{5} \mathrm{C}_{2} \mathrm{H}^{*}$ & $\mathrm{~A}_{5^{-}}$ & $\mathrm{DA}_{2}$ \\
\hline $\mathrm{H}_{2} \mathrm{CC}$ & $\mathrm{A}_{1} \mathrm{C}_{2} \mathrm{H}^{*}$ & $\mathrm{~A}_{4} \mathrm{R}_{5^{-}}$ & $\mathrm{A}_{5}$ & $\mathrm{DA}_{3}$ \\
\hline $\mathrm{C}_{4} \mathrm{H}_{4}$ & $\mathrm{~A}_{1} \mathrm{C}_{2} \mathrm{H}-$ & $\mathrm{A}_{4} \mathrm{R}_{5}$ & $\mathrm{~A}_{5} \mathrm{C}_{2} \mathrm{H}_{2} \#$ & $\mathrm{DA}_{4}$ \\
\hline $\mathrm{C}_{4} \mathrm{H}_{2}$ & $\mathrm{~A}_{1} \mathrm{C}_{2} \mathrm{H}_{2} \#$ & $\mathrm{~A}_{2-}$ & $\mathrm{A}_{5} \mathrm{C}_{2} \mathrm{H}$ & $\mathrm{DA}_{5}$ \\
\hline $\mathrm{C}_{2} \mathrm{H}_{3}$ & $\mathrm{~A}_{1} \mathrm{C}_{2} \mathrm{H}$ & $\mathrm{A}_{2}$ & $\mathrm{~A}_{5} \mathrm{C}_{2} \mathrm{H}_{2} \mathrm{U}$ & $\mathrm{DA}_{6}$ \\
\hline $\mathrm{C}_{2} \mathrm{H}_{4}$ & $\mathrm{~A}_{1} \mathrm{C}_{2} \mathrm{H}_{2} \mathrm{U}$ & $\mathrm{A}_{2} \mathrm{C}_{2} \mathrm{H}_{2} \#$ & $\mathrm{~A}_{5} \mathrm{C}_{2} \mathrm{H}^{*}$ & $\mathrm{DA}_{7}$ \\
\hline $\mathrm{C}_{4} \mathrm{H}$ & $A_{2}-X$ & $\mathrm{~A}_{2} \mathrm{C}_{2} \mathrm{H}$ & $\mathrm{A}_{6^{-}}$ & $\mathrm{DA}_{2} \mathrm{R}_{5}$ \\
\hline $\mathrm{C}_{4} \mathrm{H}_{3} \mathrm{~S}$ & $\mathrm{~A}_{1} \mathrm{C}_{2} \mathrm{H}_{3}$ & $\mathrm{~A}_{2} \mathrm{C}_{2} \mathrm{H}_{2} \mathrm{U}$ & $\mathrm{A}_{6}$ & $\mathrm{DA}_{3} \mathrm{R}_{5}$ \\
\hline $\mathrm{C}_{4} \mathrm{H}_{3} \mathrm{U}$ & $\mathrm{A}_{1} \mathrm{C}_{2} \mathrm{H}_{3} *$ & $\mathrm{~A}_{2} \mathrm{C}_{2} \mathrm{H}^{*}$ & $\mathrm{~A}_{6} \mathrm{C}_{2} \mathrm{H}_{2} \#$ & $\mathrm{DA}_{4} \mathrm{R}_{5}$ \\
\hline $\mathrm{C}_{4} \mathrm{H}_{613}$ & $\mathrm{~A}_{2} \mathrm{R}_{5^{-}}$ & $\mathrm{A}_{3^{-}}$ & $\mathrm{A}_{6} \mathrm{C}_{2} \mathrm{H}$ & $\mathrm{CH}_{2}$ \\
\hline $\mathrm{C}_{4} \mathrm{H}_{5} \mathrm{~S}$ & $\mathrm{~A}_{2} \mathrm{R}_{5}$ & $\mathrm{~A}_{3}$ & $\mathrm{~A}_{6} \mathrm{C}_{2} \mathrm{H}_{2} \mathrm{U}$ & $\mathrm{CH}_{3}$ \\
\hline $\mathrm{C}_{4} \mathrm{H}_{5} \mathrm{U}$ & $\mathrm{A}_{2} \mathrm{R}_{5} \mathrm{C}_{2} \mathrm{H}_{2} \#$ & $\mathrm{~A}_{3} \mathrm{C}_{2} \mathrm{H}_{2} \#$ & $\mathrm{~A}_{6} \mathrm{C}_{2} \mathrm{H}^{*}$ & $\mathrm{CH}_{4}$ \\
\hline $\mathrm{C}_{6} \mathrm{H}_{2}$ & $\mathrm{~A}_{2} \mathrm{R}_{5} \mathrm{C}_{2} \mathrm{H}$ & $\mathrm{A}_{3} \mathrm{C}_{2} \mathrm{H}$ & $\mathrm{A}_{7-}$ & $\mathrm{C}_{3} \mathrm{H}_{2}$ \\
\hline $\mathrm{C}_{6} \mathrm{H}_{4}$ & $\mathrm{~A}_{2} \mathrm{R}_{5} \mathrm{C}_{2} \mathrm{H}_{2} \mathrm{U}$ & $\mathrm{A}_{3} \mathrm{C}_{2} \mathrm{H}_{2} \mathrm{U}$ & $\mathrm{A}_{7}$ & $\mathrm{He}$ \\
\hline $\mathrm{A}_{1^{-}}$ & $\mathrm{A}_{2} \mathrm{R}_{5} \mathrm{C}_{2} \mathrm{H}^{*}$ & $\mathrm{~A}_{3} \mathrm{C}_{2} \mathrm{H}^{*}$ & $\mathrm{C}_{3} \mathrm{H}_{3}$ & $\mathrm{C}_{3}$ \\
\hline $\mathrm{C}_{4} \mathrm{H}_{5} \#$ & $\mathrm{~A}_{3} \mathrm{R}_{5^{-}}$ & $\mathrm{A}_{4^{-}}$ & $\mathrm{C}$ & $\mathrm{A}_{4} \mathrm{C}_{2} \mathrm{H}_{2} \#$ \\
\hline $\mathrm{C}_{6} \mathrm{H}_{7} \#$ & $\mathrm{~A}_{3} \mathrm{R}_{5}$ & $\mathrm{~A}_{4}$ & $\mathrm{C}_{2}$ & Benzene $\left(A_{1}\right)$ \\
\hline
\end{tabular}

of the various species. These concentrations, obtained at $1.2 R_{*}$, were rescaled to the gas concentration at the beginning of the hydrodynamic cooling at $1.4 R_{*}$. The initial concentration of all aromatics is set to zero.

\subsection{Results}

The following results are obtained integrating the kinetic differential equations with LSODE, a standard package for the integration of stiff differential equations using BDF methods (Gear 1971). We adopt a relative error of $10^{-4}$ (reduced, if required, during the integration) and an absolute error of $10^{-10} \mathrm{~cm}^{-3}$.

\subsubsection{PAH formation and temperature}

Figure 4 contains four representative plots of the concentration of dimers and their parent PAHs, during the very first integration, at $1.4 R_{*}$. No benzene or PAH is present at the beginning.

Comparison with the temperature profile of the gas during the hydrodynamic cooling at $1.4 R_{*}$ (Fig. 1) shows that PAH formation becomes efficient at high temperatures in a relatively high density gas, as already observed in flame experiments. Previous studies (Frenklach \& Feigelson 1989; Cherchneff et al. 1992) derived a lower temperature window (1100-900 K) for the production of PAHs to be efficient, possibly because of the lower gas densities considered in their models.

All these results are affected by the hypotheses adopted in our reactions network, in particular the growth of PAHs through linear lumping and the formation of dimers accoding to Cherchneff (1997). A consequence of these assumptions can be seen in Fig. 4. Two of the plots, one including $A_{3}$ and the other including $\mathrm{A}_{5}$, are very similar. This is because the chemistry of different PAHs is the same and the initial concentrations in this case are the same. We also point out that the high concentration of $\mathrm{A}_{7}$ corresponds to the fact that this is the biggest PAH in our model. While smaller PAHs are consumed by the growth process, this does not happen for $\mathrm{A}_{7}$. Carbon atoms "migrate" from small to big PAHs, but accumulate in $A_{7}$.

\subsection{Yields}

Rather than considering single species, it is more interesting and instructive from the point of view of dust formation to ask if the amount of carbon that is moving from simple hydrocarbons (acetylene) to PAH and dimers is enough to explain the formation of dust in IRC+10216.

To do this, the quantity "yield of aromatics [or dimers]" is usually introduced. It is defined as the ratio between the number of carbon atoms in aromatics [or dimers] and the number of carbon atoms contained in hydrocarbons. This gives the fraction of carbon that has migrated from hydrocarbons to aromatics and dimers. When speaking of the amount of $\mathrm{C}$ atoms contained in dust divided by the number of $\mathrm{C}$ atoms in hydrocarbons, we preferably use the word "fraction".

The value of the yield for aromatics (benzene, PAHs and dimers) and for dimers alone is shown in Fig. 5. These yields and their significance are discussed in the next section.

\section{Discussion}

\subsection{Are these yields sufficient to explain the formation of dust in IRC+10216?}

If we assume that dimers are the starting step in the formation of dust, an important test is to compare the amount of carbon that we have in the form of dimers, and the amount of 

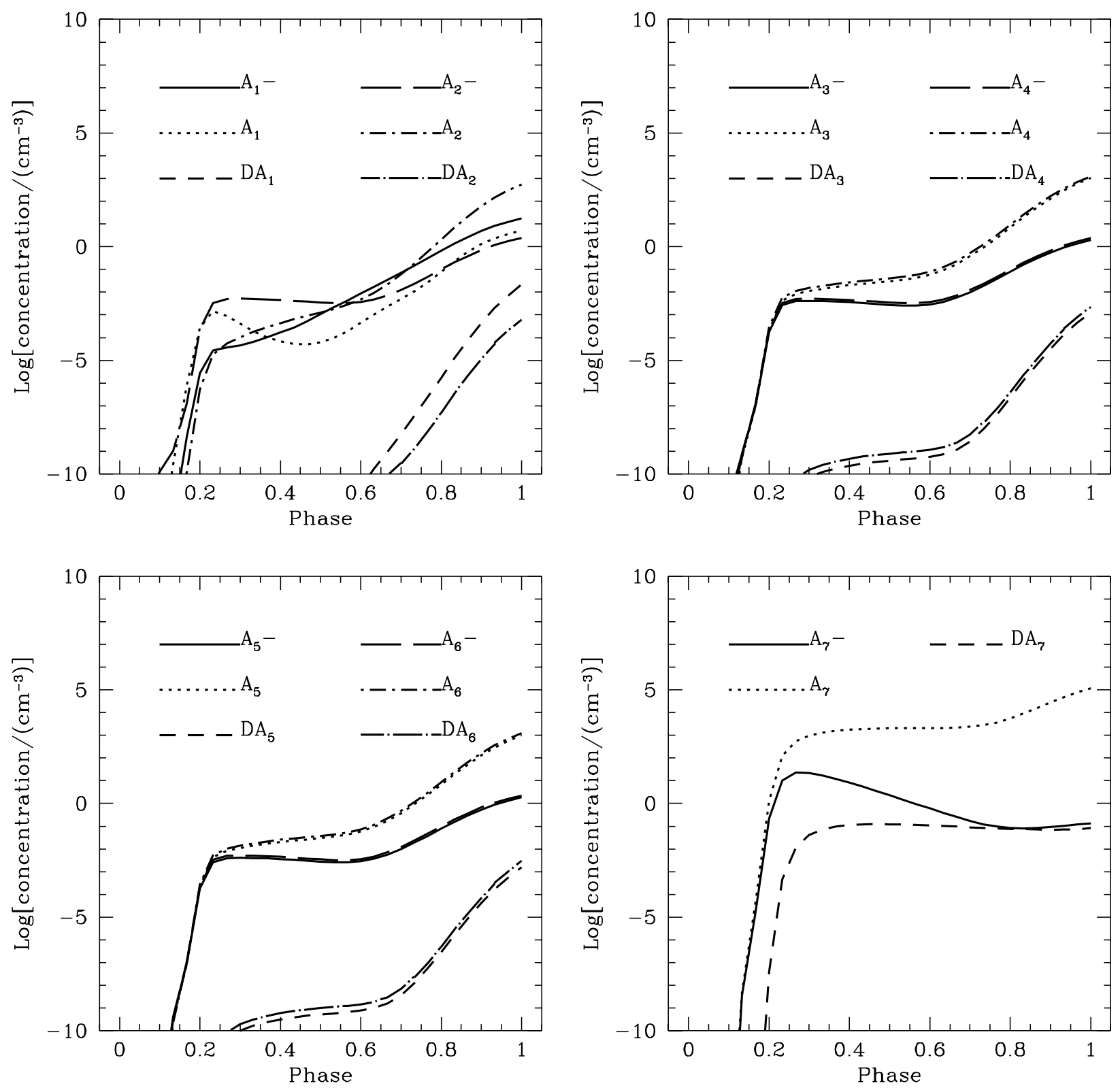

Fig. 4. Concentration of dimers and their parent molecules during the hydrodynamic cooling at $1.4 R_{*}$ (first cycle of integration). Collisional rates replace thermodynamic ones when the latter are faster.

carbon that is known to be in the form of dust in the atmosphere of carbon stars. This test needs to be done in order to check if the dimers contain at least as much carbon as the dust we expect to form from them.

For this comparison we use the ratio between the number density of the dust and the number density of the gas (dust to gas ratio) for carbon stars. This has been determined by Knapp (1985) who used 40 evolved giant stars (of which 18 were carbon stars, including IRC+10216), and derived the result that the ratio is the same (within uncertainties) in carbon-rich and oxygen-rich stars. For carbon-rich stars she obtained the value $5.2 \pm 1.7 \times 10^{-13}\left(7.6 \pm 0.4 \times 10^{-13}\right.$ for oxygen-rich stars $)$.

If we consider carbon grains with a radius of $500 \AA$ (Jura 1996), then, assuming the density for graphite grains of $1.8 \mathrm{~g} \mathrm{~cm}^{-3}$ (e.g. Cadwell et al. 1994), each of them would contain $4.7 \times 10^{7}$ carbon atoms. Considering the preshock gas density at $3 R_{*}\left(2.24 \times 10^{12} \mathrm{~cm}^{-3}\right.$, see Table 1$)$, the dust to gas ratio above implies that about $1.2 \mathrm{~cm}^{-3}$ is the actual number density of grains, so that the total amount of carbon atoms stored in grains per unit volume is $1.2 \times 4.7 \times 10^{7} \mathrm{~cm}^{-3}=5.6 \times 10^{7} \mathrm{~cm}^{-3}$. To see if molecules can provide this amount of carbon atoms, we need the total amount of carbon contained in hydrocarbons. As acetylene is the most abundant hydrocarbon, in this calculation we consider the carbon contained in it as representative of the carbon contained in all hydrocarbons. The preshock concentration of acetylene at $3 R_{*}$ (after the two cycles of integration) is $6.1 \times 10^{8} \mathrm{~cm}^{-3}$, so that the fraction needed to be incorporated into carbon dust is $5.6 \times 10^{7} / 6.1 \times 10^{8}=9.1 \times 10^{-2}$. Closer to the star, analogous calculations imply (see Table 7 for the concentration of acetylene) at $2.4 R_{*}$ a fraction of $8.9 \times 10^{-1}$, at $1.9 R_{*}$ a fraction of $1.3 \times 10^{-1}$, at $1.7 R_{*}$ a fraction of $7.9 \times 10^{-2}$ and finally, at $1.4 R_{*}$ a fraction of $9.1 \times 10^{-2}$. 
Table 7. Concentration $\left(\mathrm{cm}^{-3}\right)$ of acetylene at the end of the pulsational period at various distances considered in our model.

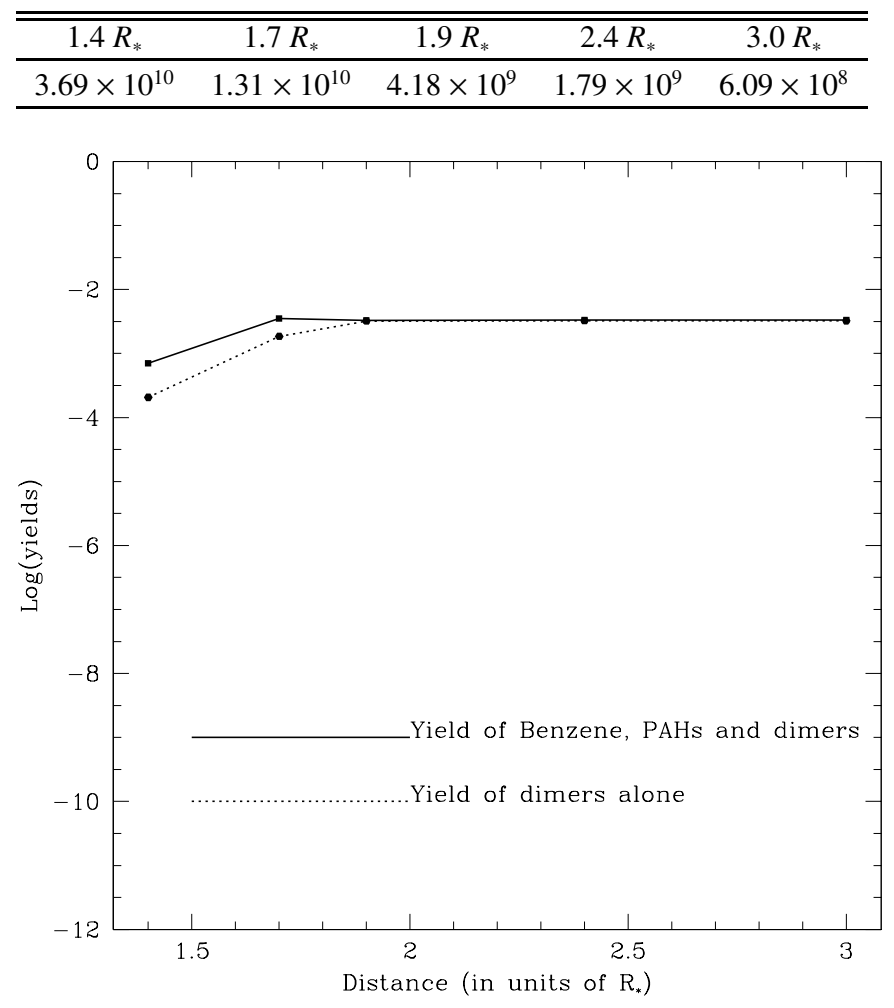

Fig. 5. Yields of benzene, PAHs and dimers, and dimers alone.

Figure 5 shows the yields for all the aromatics (PAHs, benzene and dimers), and for dimers alone. As can be seen, the yields are too low $\left(<10^{-2}\right)$ and do not compare with the fractions obtained above. That is to say that dimers and PAHs contain an amount of carbon atoms which is lower than that containedin the dust we expect to form from them.

With these very low yields it is not possible to explain the formation of grains with a radius of $500 \AA$ and the dust to gas ratio of $5.2 \pm 1.7 \times 10^{-13}$ obtained by Knapp (1985).

\subsection{Are these yields appropriate to explain the formation of nanocrystalline cores observed inside presolar grains?}

Bernatowicz et al. (1996) reported that many presolar grains contain nanocrystalline cores consisting of disorderly stacked PAH-like materials, whose diameter is in the range 5-50 $\AA$, and which are thought to have acted as condensation nuclei for the carbon. Using the simple relation $n_{\mathrm{C}} \simeq(d / 1.2)^{3}$ where $n_{\mathrm{C}}$ the number of carbon atoms contained in the grain and $d$ is its diameter in $\AA$ (the density of graphite, $1.8 \mathrm{~g} \mathrm{~cm}^{-3}$, has been assumed here) we have that the number of carbon atoms contained in these cores ranges from $2 \times 10^{2}$ to $7 \times 10^{4}$.

Consider the situation at $3 R_{*}$ where the gas number density is $2.24 \times 10^{12} \mathrm{~cm}^{-3}$ (see Table 1 ). The yield of dimers is $3.3 \times 10^{-3}$ (see Fig. 5) and the ratio between the number density of acetylene and the gas is $6.1 \times 10^{8} / 2.2 \times 10^{12}=2.8 \times 10^{-4}$, so that the ratio between the number of carbon atoms contained in dimers and the number density of the gas is $3.3 \times 10^{-3} \times$ $2.8 \times 10^{-4}=9.2 \times 10^{-7}$. Given the number of carbon atoms contained in the cores we obtained above, this corresponds to a cores to gas ratio ranging from $4.6 \times 10^{-9}$ to $1.3 \times 10^{-11}$. Following evidence from Bernatowicz et al. (1996) we assume that each core will evolve into a dust grain, so that the dust to gas ratio obtained by Knapp (1985) holds also for the cores. We can then directly compare our cores to gas ratio with the dust to gas ratio measured by Knapp. The fact that the former is significantly higher shows that while the yields are not large enough to explain the formation of the full grains, they are appropriate to explain the formation of the nanocrystalline cores observed inside grains. The same result holds closer to the star as long as both the yield and the abundance of acetylene do not change significantly (i.e., going back up to $1.9 R_{*}$ ).

\section{Conclusions}

Our chemical network does not allow to predict the concentration of single PAHs. Rather, it allows to investigate the ability of $\mathrm{C}$ atoms to "migrate" from hydrocarbons to benzene and PAHs, eventually becoming available for the formation of dust.

In the discussion we have already concluded that such migration is not as efficient as required to explain the formation of circumstellar dust if only PAHs (and their dimers) were involved, i.e., that these molecules, once formed, contain less carbon than the dust we expect to form from them.

This result has already been obtained in previous kinetic chemical reaction models (Frenklach \& Feigelson 1989; Cherchneff et al. 1992) as well as chemical pathway computations (Goeres 1993) and chemical equilibrium calculations (Helling et al. 1996). All these calculations used simple wind models which ignore the presence of shocks. Such a negative result raised the question if it was the poor wind model which was the reason for the failure of the calculations in predicting the formation of PAH molecules in an amount comparable in mass with that of circumstellar grains.

Our paper gives a negative answer to this question. Frenklach \& Feigelson (1989) found a yield that, in the most favourable case, is $1.3 \%$. This most favourable case corresponds to their "standard" model: a very low effective temperature $\left(T_{\text {eff }}=1500 \mathrm{~K}\right)$, a mass loss rate as high as $10^{-4} M_{\odot} \mathrm{yr}^{-1}$ and a wind velocity of just $10^{-2} \mathrm{~km} \mathrm{~s}^{-1}$. Cherchneff et al. (1992) obtained a yield that, in their most favourable case, was $5 \times 10^{-5}$. This case corresponds to an effective temperature of $2300 \mathrm{~K}$, a mass loss rate of $10^{-5} M_{\odot} \mathrm{yr}^{-1}$ and includes the contribution of inverse greenhouse effect to the temperature of PAHs. The yield of PAHs and dimers we obtain is, at the most, $3.3 \times 10^{-3}$. This is lower than that found by Frenklach $\&$ Feigelson (1989) and though it is two orders of magnitude larger than that obtained by Cherchneff et al. (1992), it is still too small to explain the formation of carbon grains if PAHs are their only building blocks. However, carbon does not need to be locked into PAHs to become available for dust formation, because a significant percentage of carbon can be incorporated in the grain through deposition of hydrocarbons, particularly acetylene, onto its surface. While PAHs are required for the 
initial nucleation of carbon grains, they are not required for their growth.

Of course other models which take shocks into account could provide a different answer to the question. In addition, we have adopted only a certain set of parameters for our model of the circumstellar envelope (stellar mass, density and effective temperature, $\mathrm{C} / \mathrm{O}$ ratio, shock velocity) and a different set of values may result in very different yields.

Nevertheless, in the discussion we have shown that the amount of carbon that "migrates" into dimers can readily account for the formation of the nanocrystalline cores found in presolar grains.

Another conclusion of this paper is that the formation of PAHs is efficient at higher temperature than predicted in previous models. A possible explanation of this fact is that the higher gas number density in our model makes the chemistry more efficient.

Acknowledgements. I would like to thank Isabelle Cherchneff, Tom Millar, Debiprosad Duari and Yoann Le Teuff for many discussions on the subject of dust formation in circumstellar environments and Andrew Markwick for having provided his program CHEMEQ. I also acknowledge a scholarship from Regione Autonoma della Sardegna.

\section{Appendix A: Temperature and concentration profiles}

\section{A.1. Preshock temperature and concentration of the gas}

We assume that the radial temperature profile is represented by (e.g. Bowen 1988)

$T(r)=T_{\text {eff }}\left(\frac{r}{R_{*}}\right)^{-0.6}$.

The concentration profile is determined as in Cherchneff et al. (1992).

The damping of the shock at increasing radius is determined using a result by Willson \& Bowen (1984)

$v_{\text {shock }}(r)=v_{\text {shock }}\left(r_{\text {shock }}\right)\left(\frac{r}{r_{\text {shock }}}\right)^{-0.5}$.

We can now consider the problem of how the physical parameters change when a shock passes through a parcel of gas. To handle this problem we distinguish three different stages. Firstly, the parcel is compressed and heated by the shock front, secondly it cools through the dissociation of $\mathrm{H}_{2}$ and when this process becomes less efficient than the hydrodynamic cooling, the adiabatic expansion begins and the parcel of gas is accelerated upwards and falls then back under the influence of stellar gravity.

\section{A.2. Temperature and concentration of the gas behind the shock front}

We assume that the rapid variations of concentration and temperature occurring in a parcel of gas when the shock front reaches it are well described by the well known RankineHugoniot conditions which relate the thermodynamic variables before and after a shock front when inelastic collisions are not included. The analytical expressions (e.g. Nieuwenhuijzen et al. 1993) for temperature and concentration are

$$
\begin{aligned}
& \frac{T_{1}}{T_{0}}=\frac{\left[2 \gamma M^{2}-(\gamma-1)\right]\left[(\gamma-1) M^{2}+2\right]}{(\gamma+1)^{2} M^{2}} \\
& \frac{n_{1}}{n_{0}}=\frac{(\gamma+1) M^{2}}{(\gamma-1) M^{2}+2}
\end{aligned}
$$

where the index 0 denotes the preshock gas and 1 the gas immediately after the shock front. $M=v_{\text {shock }} / a$ is the Mach number, where $a$ is the sound velocity, and $\gamma=c_{\mathrm{p}} / c_{\mathrm{v}}$ is the adiabatic exponent for which we assume the ideal, diatomic gas value of $\gamma=1.4$, which is accurate to within 5 percent for most diatomic molecules, and is nearly exact for our main gas, $\mathrm{H}_{2}$. The temperature and concentration at the shock front are reported, at various radii, in the sixth and seventh columns of Table 1.

\section{A.3. Cooling processes}

Once the gas is heated and compressed by the shock, it cools via various microscopic processes and via work, i.e., expansion. To model these processes we introduce a simplification and distinguish two stages, corresponding to which process dominates the cooling. A first stage in which only microscopic processes are responsible for cooling the gas (chemical cooling) and a following stage in which only expansion is responsible for this (hydrodynamic cooling).

\section{A.4. "Chemical" cooling of the gas}

Fox \& Wood (1985) have shown that for cool, molecular preshock gas, with values of concentration and shock velocities assumed in our model (see model B20 in their paper), radiative cooling is not efficient because only collisions with atoms (not with electrons) are responsible for ionization and excitation and the resultant emission intensities are very low. On the contrary they found that the collisional dissociation of molecular hydrogen by atomic hydrogen

$\mathrm{H}+\mathrm{H}_{2} \rightarrow 3 \mathrm{H}$

is very efficient in cooling the gas, and we model the thermal cooling as if only this process were responsible for cooling the gas, following Willacy \& Cherchneff (1998). We assume that the chemical cooling lasts until the parameters have reached the values where the hydrodynamic cooling begins (values determined as explained in Sect. A.6). A logarithmic fitting of the parameters between the shock front values and the values at the beginning of the hydrodynamic cooling is used to describe their variation during the chemical cooling. The length of the thermal cooling layer is defined (Willacy \& Cherchneff 1998) by the collisional dissociation of molecular hydrogen by atomic hydrogen whose rate in $\mathrm{cm}^{3} \mathrm{~s}^{-1}$ is (Willacy \& Cherchneff 1998)

$$
k=3.78 \times 10^{-8}\left(\frac{T}{300}\right)^{-0.5} \exp \left(\frac{-53280 \mathrm{~K}}{T}\right)
$$


where $T$ is the kinetic temperature in $\mathrm{K}$. The $\mathrm{H}_{2}$ dissociation length $l_{\mathrm{D}}$ is defined as

$l_{\mathrm{D}}=\frac{1}{k n_{\mathrm{H}}} \frac{v_{\text {shock }}}{n_{\text {jump }}}$

where $n_{\mathrm{H}}$ is the number density of atomic hydrogen, $v_{\text {shock }}$ is the shock velocity in the stellar rest frame and $n_{\text {jump }}$ is $n_{1} / n_{0}$ in Eq. (A.4). Figures 1 and 2 show (left) the temperature and gas concentration during the chemical cooling at two different distances considered in our model. The values of $l_{\mathrm{D}}$ used in these plots reflect typical values found in the calculations presented in this paper.

\section{A.5. Hydrodynamic cooling of the gas}

We consider here the effect of expansion as described by Bowen (1988), Bertschinger \& Chevalier (1985) and Willacy \& Cherchneff (1998). Parcels of gas are pushed up because of the expansion and fall then back because of stellar gravity. We adopt the analytical treatment of the hydrodynamics developed by Bertschinger \& Chevalier (1985) who solve for the continuity, momentum and energy equations of a periodically shocked, adiabatically expanding gas subjected to the stellar gravitational field. As required by this analytical treatment, we assume that when the parcel falls back it reaches the same position and same physical conditions it had before the shock reached it. At that point it is shocked again and the cycle repeats itself continuously. In more realistic treatments the parcel of gas never comes back to the initial position but it ends the excursion at progressively increasing distance from the star, giving rise to a net wind (Bowen 1988). Nevertheless, the approximation of parcels of gas falling back to their initial position is appropriate for the regions near the photosphere (Bowen 1988).

\section{A.5.1. Temperature}

In the analytical treatment of Bertschinger \& Chevalier (1985) the temperature during the expansion is written as

$T=\frac{\mu u^{2}}{k} \theta$

where $\mu$ is the mean molecular mass, $u$ is the velocity of the gas in the shock frame and $k$ is the Boltzmann constant. Here $\theta$ is a non-dimensional quantity that can be written as

$\theta=\frac{1}{\gamma M^{2}}\left(\frac{M^{\frac{2}{\gamma+1}}}{w}\right)^{(\gamma-1)}$

where $w$ is in the interval $\left(w_{0}, M^{\frac{2}{\gamma+1}}\right), M$ being the Mach number. For $w_{0}$ see Sect. A.6. The model does not give an expression for $\theta$ as a function of the phase, but it is nevertheless possible to determine the value of the phase for each adopted value of $w$. For each given value of $w$, we find both $\theta$ and the phase, and then fit $\theta$ as a function of the phase. To implement this, we consider $w=\left(0.1,0.2,0.3, \ldots, M^{\frac{2}{\gamma+1}}\right)$ and use a polynomial of fourth degree to perform the fit. Figures 1 and 2 show the resulting fit for the temperature (A.8) (top of the figures) of a parcel of gas at two different distances from the star. It is worth noticing that the gas cools below its radiative equilibrium temperature (fourth column in Table 1), a result first mentioned by Bowen (1988).

\section{A.5.2. Concentration}

In Bertschinger \& Chevalier (1985) the mass density of the gas $\rho$ is written in the form

$\rho=\rho_{0} D$

where $\rho_{0}$ is the mass density of the gas before the shock arrives. The non-dimensional quantity $D$ can be written as

$D=\frac{M^{\left(\frac{2}{\gamma+1}\right)}}{w}$

and, as for the temperature, this allows us to determine the mass density $\rho$ and the phase for different values of $w$, and to fit $\rho$ as a function of phase. The same values of $w$ were used, and again a fourth degree polynomial was used for the fit.

Given the mass density of the gas, its concentration is known once we know its chemical composition. Since the chemical composition changes with time, the concentration of the gas does not evolve in the same way as the mass density, particularly because of reaction (A.5). To determine the concentration of each species during the excursion, we consider the contribution of both the expansion of the gas and the chemistry. This involves the use of the equation of continuity and of chemical kinetic equations.

Figures 1 and 2 show the concentration of the gas during the expansion assuming a constant chemical composition.

\section{A.6. Determination of the values of concentration and temperature at which the expansion begins}

The value of the concentration of the gas at which the chemical cooling ends and the hydrodynamic cooling begins is given by $n_{\mathrm{c} / \mathrm{h}}=n_{0} D_{\mathrm{c}}$, where $n_{0}$ is the preshock concentration and $D_{\mathrm{c}}$ is the solution of Eq. (2.21) in Bertschinger \& Chevalier (1985),

$D_{\mathrm{c}}^{\gamma+1}-\left(1+\gamma M^{2}\right) D_{\mathrm{c}}+\gamma M^{2}=0$.

Using our knowledge of the concentration as a function of $w$, we can determine the value $w_{0}$ at which the gas reaches concentration $n_{\mathrm{c} / \mathrm{h}}$ and the corresponding temperature $T_{\mathrm{c} / \mathrm{h}}$. In this way we determine the values of the concentration and temperature of the gas when the chemical cooling ends and the hydrodynamic cooling begins. The values of $T_{\mathrm{c} / \mathrm{h}}$ and $n_{\mathrm{c} / \mathrm{h}}$ are reported in the eight and ninth columns in Table 1.

\section{References}

Allain, T., Sedlmayr, E., \& Leach, S. 1997, A\&A, 323, 163

Avery, H. E. 1974, Basic Reaction Kinetics and Mechanism (London, Macmillian)

Bernatowicz, T. J., Cowsik, R., Gibbons, P. C., et al. 1996, ApJ, 472, 760

Bertschinger, E., \& Chevalier, R. A. 1985, ApJ, 299, 167 
Bowen, G. H. 1988, ApJ, 329, 299

Cadwell, B. J., Wang, H., Feigelson, E. D., \& Frenklach, M. 1994, ApJ, 429, 285

Chase, M. W., Davies, C. A., Downey, J. R., et al. 1985, J. Phys. Chem., Ref. Data 14, Suppl. 1, 1

Cherchneff, I., Barker, J. R., \& Tielens, A. G. G. M. 1991, ApJ, 377, 541

Cherchneff, I., Barker, J. R., \& Tielens, A. G. G. M. 1992, ApJ, 401, 269

Cherchneff, I. 1997, in Molecules in Astrophysics: Probes and Processes, IAU 1997, ed. van Dishoeck, 469

Fahr, A., Mallard, W. G., \& Stein, S. E. 1988, in 21st Symp. (Int.) on Combustion, The Combustion Institute, Pittsburgh, 825

Fox, M. W., \& Wood, P. R. 1985, ApJ, 297, 455

Frenklach, M., Clary, D. W., Gardiner, W. C., \& Stein, S. E. 1985, in 20th Symp. (Int.) on Combustion, The Combustion Institute, Pittsburgh, 887

Frenklach, M., Clary, D. W., Gardiner, W. C., \& Stein, S. E. 1986a, in Proc. 15th Int. Symp. Shock Waves and Shock Tubes, ed. D. Bersader, \& R. Hanson (Sanford: Stanford University Press), 295

Frenklach, M., Clary, D. W., Yuan, T., Gardiner, W. C. Jr., \& Stein, S. E. 1986b, Combustion Sci. Tech., 50, 79
Frenklach, M., \& Feigelson, E. D. 1989, ApJ, 341, 372

Gear, W. C. 1971, Numerical Initial Value Problems in Ordinary Differential Equations (Prentice Hall)

Gillet, D., Maurice, E., \& Baade, D. 1983, A\&A, 128, 384

Goeres, A. 1993, in Rev. Mod. Astron., ed. G. Klare, 6, 164

Helling, C., Jørgensen, Pletz, B., \& Johnson, H. R. 1996, A\&A, 315, 194

Jura, M. 1996, in Astrophysical Implications of the Laboratory Study of Presolar Materials, ed. T. J. Bernatowicz, \& E. Zinner (St. Louis), 379

Knapp, G. R. 1985, ApJ, 293, 273

Miller, J. H., Mallard, W. G., \& Smyth, K. C. 1984, J. Phys. Chem., 88,4963

Nieuwenhuijzen, H., de Jager, J., Cuntz, M., Lobel, A., \& Achmad, L. 1993, A\&A, 280, 195

Sharp, C. M., \& Huebner, W. F. 1990, ApJ, 72, 417

Stein, S. E., \& Fahr, A. 1985, J. Phys. Chem., 89, 3714

Willacy, K., \& Cherchneff, I. 1998, A\&A, 330, 676

Willson, L. A., \& Bowen, G. W. 1984, in Relation between Chromospheric-Coronal Heating and Mass Loss in Stars, ed. R. Stalio, \& J. B. Zirker (Trieste), 127 